\title{
Correction-free remotely scanned two-photon in vivo mouse retinal imaging
}

\author{
Adi Schejter Bar-Noam, Nairouz Farah and Shy Shoham
}

Non-invasive fluorescence retinal imaging in small animals is an important requirement for an array of translational vision applications. The in vivo two-photon imaging of the mouse retina may enable the long-term investigation of the structure and function of healthy and diseased retinal tissue. However, to date, this has only been possible using relatively complex adaptive-optics systems. Here, the optical modeling of the murine eye and of the imaging system is used to achieve correction-free two-photon microscopy through the pupil of a mouse eye to yield high-quality, optically sectioned fundus images. By remotely scanning the focus using an electronically tunable lens, high-resolution three-dimensional fluorescein angiograms and cellular-scale images are acquired, thus introducing a correction-free baseline performance level for two-photon in vivo retinal imaging. Moreover, the system enables functional calcium imaging of repeated retinal responses to light stimulation using the genetically encoded indicator, GCaMP6s. These results and the simplicity of the new add-on optics are an important step toward several structural, functional, and multimodal imaging applications that will benefit from the tight optical sectioning and the use of near-infrared light.

Light: Science \& Applications (2016) 5, e16007; doi:10.1038/lsa.2016.7; published online 1 January 2016

Keywords: angiography; eye model; functional calcium imaging; in vivo; optogenetics; two-photon microscopy

\section{INTRODUCTION}

Non-invasive fluorescence fundus imaging is an important tool for in vivo small animal retinal imaging in a wide array of translational vision applications. These include the tracking of fluorescently tagged cells and blood vessels over time, and the functional fluorescence imaging of calcium probes that are used to monitor retinal neuronal activity. Invariably, these various in vivo applications require systems that are capable of imaging fine retinal structures. High-resolution funduscopy primarily uses scanning laser ophthalmoscopes (SLOs) with or without adaptive optics $(\mathrm{AO})^{1-5}$ or wide field imaging through low-numeric aperture (NA) objectives ${ }^{6}$ and topical endoscopes ${ }^{7,8}$.

Using two-photon excitation for in vivo retinal imaging in small mammalian eyes has multiple potential advantages. First, the current one-photon systems use visible wavelengths to excite fluorescent markers that lead to strong light adaption ${ }^{9}$, whereas near-infrared (NIR)based two-photon excitation has been successfully utilized to obtain functional imaging of visual responses to visible light in isolated retinas ${ }^{10-13}$. Second, two-photon excitation provides an inherent optical sectioning capability, eliminating the need for additional spatial light filtering. Third, two-photon excitation autofluorescence utilizing endogenous fluorophores has been demonstrated in retinal explants $^{14-16}$ and has the advantage of reduced phototoxicity and absorption in the optics of the eye compared with ultraviolet (UV)based autofluorescence. Despite these multiple potential advantages and the widespread availability of two-photon microscopes, twophoton microscopic imaging through a mouse pupil was only recently demonstrated using a relatively complex, specialized optical system for adaptively compensating for the aberrations of the eye $\mathrm{e}^{17,18}$. Moreover, it is commonly assumed that major barriers have hindered other attempts to directly implement such imaging, such as the strong aberrations in mouse eyes ${ }^{19}$.

Here, we use a model-based approach to analyze the requirements for an in vivo two-photon retinal imaging system, and found that the mouse's eye strongly constrains the range of imaging lens combinations that can be used for this application. Next, we show that a system that follows these design criteria yields fundus images of optically sectioned, well-resolved, and fluorescent microstructures down to the cellular level (see Schejter et al. ${ }^{20}$ for preliminary reports of this work). To overcome the scan range limitations and the risk of corneal compression, our system integrates offset optics before the objective lens. This design approach is further facilitated by the insertion of a remote depth-scanning solution based on an electronically tunable lens (ETL), which was first applied in multiphoton microscopy by Grewe et al. ${ }^{21}$ The system provides major advantages in comparison with wide field imaging systems, and it allows long-term repeated imaging as well as functional calcium imaging via a simple and accessible design. The performance characteristics of this approach provide a salient and correction-free baseline to which AO two-photon systems should be compared.

\section{MATERIALS AND METHODS}

Experimental setup

The two-photon imaging system uses a Mai-Tai eHP laser tuned to $800 \mathrm{~nm}$ for the structural imaging and tuned to $920 \mathrm{~nm}$ for the functional imaging (Spectra-Physics, Santa Clara, CA, USA; available 
tuning range: $650-1040 \mathrm{~nm}$ ) with a DeepSee module that provides optimized pre-chirping for the dispersion of the optical system. To scan axially without requiring the objective to come into contact with the cornea of the animal, a convex ETL (EL-C-10-30-VIS-LD, Optotune AG, Dietikon, Switzerland), and a concave offset lens ( -100 or $-50 \mathrm{~mm}$, plano-concave, Thorlabs, Newton, NJ, USA) were positioned in front of a $10 \times$ water immersion objective (Zeiss, Oberkochen Germany, $0.45 \mathrm{NA}$, WD $=1.8 \mathrm{~mm}$ or Nikon, Melville, $\mathrm{NY}, \mathrm{USA}, 0.3 \mathrm{NA}, \mathrm{WD}=3.5 \mathrm{~mm}$ ). The objective lens was positioned horizontally and coupled to the eye while the animal faced sideways (Figure 1a; alternatively, the objective was vertical and the eye of the animal was facing upwards - not shown). The laser beam exited the objective lens $(10 \times, 0.3 \mathrm{NA})$ via a front aperture with a diameter of $4.7 \mathrm{~mm}$, which slightly overfilled the dilated pupil of the mouse ( 2 $\mathrm{mm}$ diameter). The focal distance of the ETL was tuned by applying a control current between $0 \mathrm{~mA}$ and $300 \mathrm{~mA}$ and using a current driver that scans at $0-2000 \mathrm{~Hz}$ (Electrical Lens Driver 4, Optotune AG, Dietikon, Switzerland). The emitted light was reflected off a dichroic mirror (FF685-Di02, Semrock, Rochester, NY, USA) and filtered using an infrared (IR) blocking short-pass filter (E680SP-2P) and a custom bandpass filter (525/80 nm, Omega Optical, Brattleboro, VT, USA) to block out light stimulation during the functional imaging. Both filters were placed before the photomultiplier tube (PMT, H9307, Hamamatsu, Japan). For the functional imaging, light from an epi-fluorescence illuminator (Intensilight, Nikon, Melville, NY, USA) was collimated, passed through a rapid shutter ( $\mathrm{SH} 05$ with a SC10 shutter controller, Thorlabs, Newton, NJ, USA), filtered for blue light (HQ480/40×, Chroma, Bellows Falls, VT, USA) and reflected off a long-pass dichroic mirror (490dcxr, Chroma, Bellows Falls, VT, USA), which was chosen for its high transmission in the GCaMP emission spectra. For comparative one-photon imaging, we used a Topical Endoscopy Fluorescence Imaging (TEFI) system ${ }^{8}$ that combines an otoscope (Karl Storz Endoscopy, Tuttlingen, Germany) and a digital single-lens reflex camera (D5000, Nikon, Melville, NY, USA).

\section{Optical models}

We constructed two optical models of the compound optical system (Figure 1b): (1) a simplified, analytically tractable paraxial optical model and (2) a detailed ray tracing-based model that was implemented in the Zemax software (ZEMAX Development Corporation, Kirkland, WA, USA) used to validate the paraxial results and to provide imaging performance analysis. For the paraxial model (Figure 1b, top), each of the optical elements was represented by a thin lens with the respective focal distance and location, whereas the ocular optics were modeled as two thin lenses (for the cornea and crystalline lens) whose focal distances $(f=4.5 \mathrm{~mm}$ and $2.05 \mathrm{~mm} @$ $488 \mathrm{~nm}$ ) were obtained from Remtulla et al. ${ }^{22}$ The crystalline lens location of the mouse was calibrated such that the eye focused the collimated visible light onto the outer retina $\left(d_{\text {cornea-lens }}=2.35 \mathrm{~mm}\right)$, and its focal distance was extrapolated from the measured visible range $^{22}$ to the $800 \mathrm{~nm}$ imaging wavelength $\left(f_{800 \mathrm{~nm}}=2.25 \mathrm{~mm}\right)$. During imaging, the refraction of the cornea was canceled due to the index matching with the coupling saline solution. The respective ray transfer matrices for each lens and free space propagation were multiplied. By constraining the vector at the focal plane to be dependent only on the galvo mirror angle (independent of the position of the ray in the galvo plane), we derived the relations between the various optical parameters.

For the ray-tracing-based modeling, we used Zemax to analyze the detailed optical model of the near-eye optics (Figure 1b, bottom). The concave offset lens (CO, $-100 \mathrm{~mm}$ ) and the ETL's Zemax data were provided by the respective companies, whereas the $10 \times 0.3 \mathrm{NA}$ objective lens was modeled with modifications from a patent a

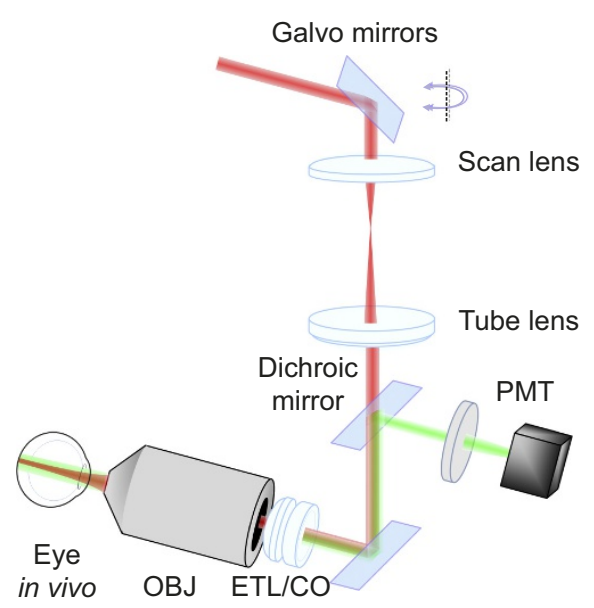

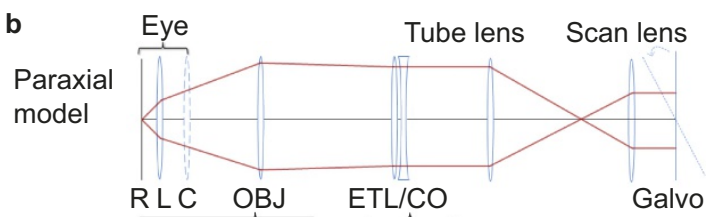

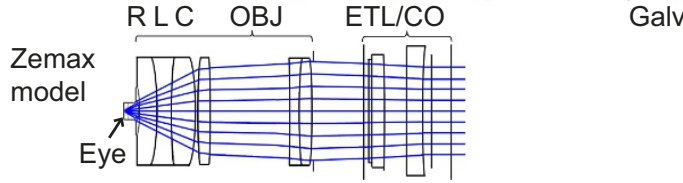

C

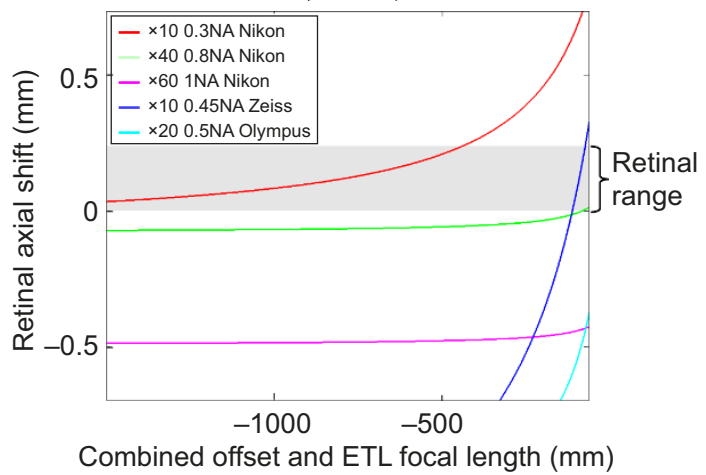

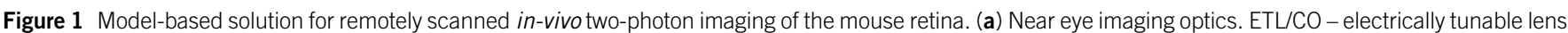

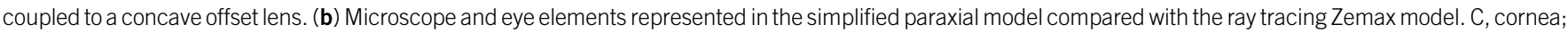

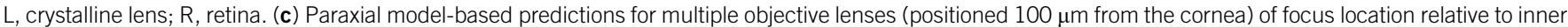
limiting membrane plane as a function of the combined offset focal length (shaded area-retinal range). 
(US6128139, modified to our NA and working distance), and the eye was modeled according to the mean parameters found in Remtulla et al. $^{22}$ The one-photon point spread function (PSF) analysis was performed on rays at the center of the focal (maximal intensity) plane using the Zemax Huygens PSF analysis, and the two-photon PSFs were estimated from these using Matlab.

\section{Mouse in vivo experiments}

During the imaging experiments, we used an adjustable clamp to correctly position the eye and minimize the head motion of the mouse by securing a metal bar that was surgically fixed to the skull of the C57Bl adult mice (8-16 weeks). The pupils were dilated with eye drops ( $1 \%$ atropine sulfate, $0.5 \%$ tropicamide, $2.5 \%$ phenylephrine), and a saline solution $(\mathrm{NaCl} 0.9 \%)$ was used to keep the eye hydrated and coupled with the water immersion objective. The mice were anesthetized by an intraperitoneal (IP) injection of ketamine $\left(50 \mathrm{mg} \mathrm{kg}^{-1} \mathrm{BW}\right)$ and medetomidine $\left(1 \mathrm{mg} \mathrm{kg}^{-1} \mathrm{BW}\right)$, and $20 \mu \mathrm{L}$ of $10 \%$ fluorescein (Sigma-Aldrich, St. Louis, MO, USA) was injected prior to the imaging.

Six 5-week-old mice were intravitreally injected with an adenoassociated virus (AAV) that expressed the genetically encoded calcium indicators, GCaMP3 ${ }^{23}$ and GCaMP6 ${ }^{24}$. GCaMP3 was used for the structural imaging due to its higher baseline fluorescence, whereas GCaMP6s was used for the functional imaging because of its larger $\mathrm{dF} / \mathrm{F}$. Two mice were injected with AAV2/1.hSynap.GCaMP3.3.SV40, and four were injected with AAV1.Syn.GCaMP6s.WPRE.SV40 (University of Pennsylvania Vector Core, Philadelphia, PA, USA). Approximately, $1 \mu \mathrm{L}$ of the virus was injected using a 31 gauge blunt needle (Hamilton, Bonaduz, Switzerland). The mice were imaged 3-8 weeks following the injection. In two of the GCaMP6s-injected mice, we clearly visualized the transfected cells, and these were used for the functional imaging.

For the functional imaging experiments, the duration and timing of the wide-field flashes $\left(16 \mathrm{~mW} \mathrm{~cm}^{-2}\right.$ at the pupil) were controlled by triggering the mechanical beam shutter from the two-photon scanning software.

The animal experiments were conducted in accordance with the Institutional Animal Care Committee at the Technion - Israel Institute of Technology.

\section{Image analysis}

The raw images were acquired using ScanImage software ${ }^{25}$ with a resolution of $512 \times 512$ pixels and $2 \mathrm{~ms}$ per line (imaging rate of $\sim 1$ fps). The images were de-noised, and the contrast was enhanced using image processing tools for optimal visualization (ImageJ and Adobe Photoshop). All of the calculations were based on data extracted from the raw images (using Matlab). The in vivo lateral scan range was estimated from the relative changes in the optic disc size across different axial cross-sections. To estimate the lateral imaging resolution of the system, we created an average cell image (by aligning $n=7$ 'doughnut' shaped cells) from which we extracted a profile as a function of the radial distance from the center of the cell. The same analysis was performed to a set of high-resolution in vitro two-photon retinal images, and the width of both profiles was used to estimate the lateral resolution using a Gaussian shape assumption:

$$
\sigma_{\text {invivo }}^{2}=\sigma_{\text {invitro }}^{2}+\sigma_{P S F}^{2}
$$

Functional imaging movies were acquired at a low resolution of $128 \times 128$ pixels and $1 \mathrm{~ms}$ per line (imaging rate of $\sim 6.7 \mathrm{fps}$ ) or $128 \times 64$ pixels and $4 \mathrm{~ms}$ per line $(3.4 \mathrm{fps})$. Following the elimination of 2-3 frames that were contaminated by the stimulus artifact, the fluorescence signals were calculated from $\frac{d F}{F}(t)=\frac{F(t)-F_{0}}{F_{0}}$, where $F_{0}$ is the average baseline signal of each cell and $F(t)$ is its temporal fluorescence signal (both dark current subtracted), and then the signals were bandpass filtered $(0.05-0.7 \mathrm{~Hz})$. The mean response for each cell was calculated by averaging the individual responses per stimulus, which was fitted with an exponential decay function using a minimum mean square error (MMSE) criterion.

\section{RESULTS AND DISCUSSION}

The ray-transfer matrices of the model were used to analyze the expected focal position inside the eye using several commercially available objectives (and their combinations with appropriate ETL and concave offset lenses, together referred to as 'combined offset and ETL' in Figure 1c). For example, we determined the distance between the inner limiting membrane plane and the imaging plane $\left(d_{\text {retina-focus }}\right)$ as a function of the combined concave offset and the ETL focal length, $\left(f_{\mathrm{ETL}}+\mathrm{CO} \approx \frac{f_{\mathrm{ETL}} \cdot f_{\mathrm{CO}}}{f_{\mathrm{ETL}}+f_{\mathrm{CO}}}\right)$, to obtain the following relation (Figure $1 \mathrm{c}$, red line, $10 \times 0.3 \mathrm{NA}$ objective):

$$
d_{\text {retina-focus }}=\frac{51.2+0.023 * f_{\mathrm{ETL}+\mathrm{CO}}}{42.5-0.29 * f_{\mathrm{ETL}}+\mathrm{CO}}
$$

and the variables and constants in the equation are in millimeters.

This analysis (Figure 1c) showed that the vast majority of available water-dipping objectives will be focused by the crystalline lens in front of the retina even when the objective comes in contact with the cornea; the only exception in our set were the low-magnification $10 \times$ objectives from Zeiss (Oberkochen Germany, 0.45NA, WD $=1.8 \mathrm{~mm}$ ) and from Nikon (Melville, NY, USA, 0.3NA, WD = $3.5 \mathrm{~mm}$ ), and the latter provided a much wider working range and a superior ease of use. Indeed, we were unable to image the retina except when using these objectives, validating this result (see below for validation).

To validate the accuracy and agreement between the optical models, we compared the model-based predictions both inside and outside of the eye, including the dependence on the ETL focal distance of the axial shift (Figure 2a) and the lateral scan range (Figure 2b, defined as the lateral displacement per scan angle). To experimentally validate the models, we compared the model predictions of the axial shifts to the fluorescent micro-bead measurements (Figure 2a left, possible only outside the eye) and of the lateral scan range inside the eye (Figure 2b), and both demonstrated good agreement. An interesting finding from this analysis is that the axial shifts inside the eye are $\sim 4.4$ times smaller than the corresponding shifts outside the eye because of an effect introduced by the crystalline lens. Next, we used the Zemax model to analyze the expected one- and two-photon PSFs inside the eye for multiple axially shifted foci. The analysis shows that the twophoton PSF widths vary slightly as the focal plane moves away from the objective lens ${ }^{21}$ (Figure 2c), and they also remain relatively constant with increasing lateral distance from the center of the retina (Figure $2 c$, inset). The detailed axial dependence of both the one- and two-photon focal intensity distributions is further demonstrated in Figure 2d. Notably, the nonlinearity of the two-photon excitation has a major effect of reducing the PSF side lobes (also see the three-dimensional (3D) PSF in Supplementary Fig. S1). However, there are multiple eyerelated aberrations ${ }^{19}$ that we are not modeling; therefore, we are likely underestimating the experimental PSFs (our Zemax simulations only account for basic aberrations introduced by the ETL ${ }^{21}$ and the rest of the optical model). 
a

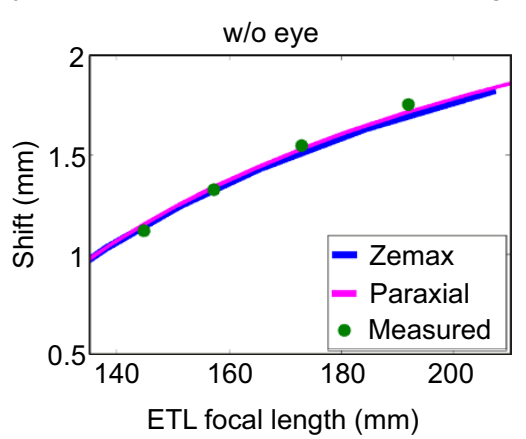

Remote axial shift

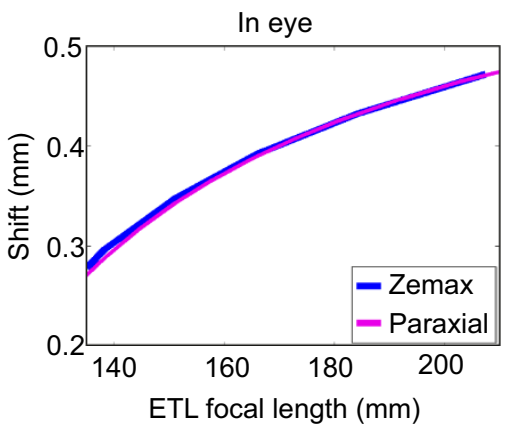

b

Scan range in eye

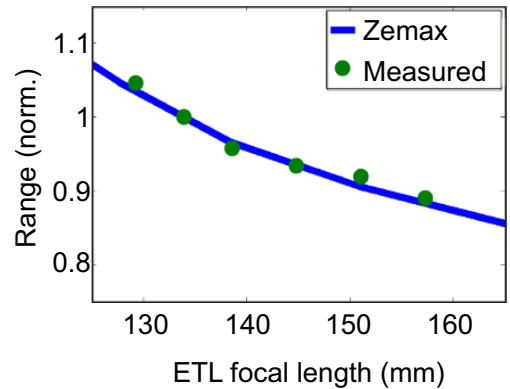

c

Predicted resolution in eye

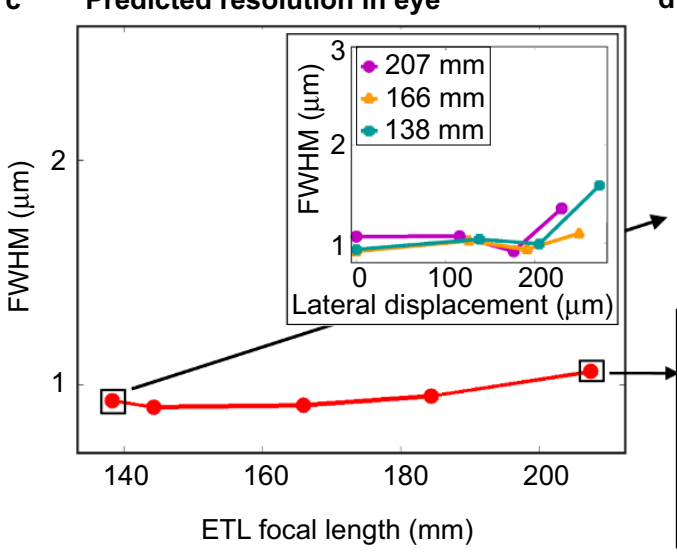

d $\quad 138 \mathrm{~mm}$

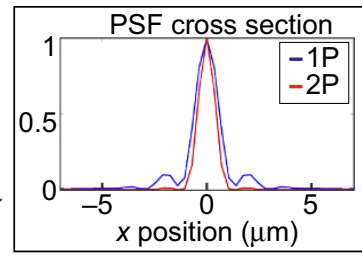
$207 \mathrm{~mm}$

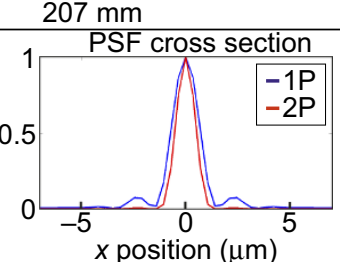

Two-photon
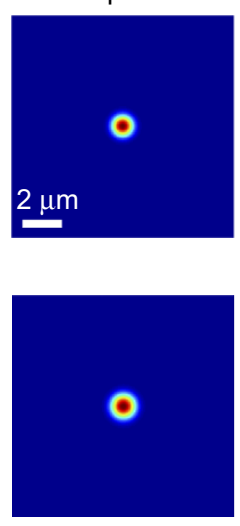

One-photon
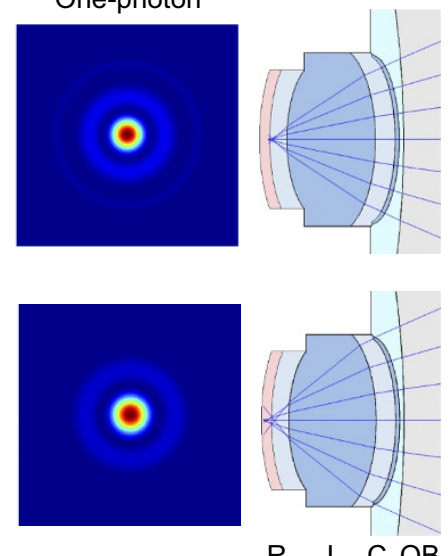

$R \quad L \quad C O B J$

Figure 2 Model validation and resolution derivation. (a) Comparison of the remote axial shift following changes for the ETL focal length between the simulated Zemax (blue) and paraxial (pink) models and the measured results (green) without an eye (left), and inside one (right). (b) In vivo comparison of the normalized scan range between the Zemax model and measured results from the retinal sections in Figure 3a. (c) Width (FWHM) of the axially shifted Zemax-simulated 2P PSFs at the center of the FOV. Inset: the PSF widths for three different ETL focal lengths (138, 166, and $207 \mathrm{~mm}$ ) as a function of the lateral distance from the center of the FOV. (d) Simulated 1P and 2P PSFs (center) and their cross-sections (left) for two different ETL focal lengths. Right: enlarged view of the eye region from the Zemax model. C, cornea (dark blue); L, crystalline lens; OBJ, objective lens (gray); 1P, one-photon; R, retina (pink); 2P, two-photon.

Next, we tested the ability of the system to acquire optically sectioned images of fluorescently labeled retinal blood vessels in vivo through the dilated pupils of anesthetized mice, and we acquired images at different depths by changing the ETL current. In each image (Figure 3a), it is possible to identify different blood vessels in the surrounding area of the optic disc, and image stacks can be used to provide a comprehensive $3 \mathrm{D}$ view of the retinal vessel network (Figure 3b). The effective fluorescent field-of-view (FOV) diameter, which was estimated for each cross section in the retinal 3D stack (Figure 3a), decreases significantly faster than the lateral scan range for the increasing axial shifts (Figure 3c) putatively due to the decreasing circumference of the retina.

To characterize the axial scanning, we repeatedly refocused the objective lens onto a selected plane of vasculature using a motorized $\mathrm{z}$-stage for multiple ETL control values. These measurements provide a strong validation of the predictions of the paraxial model (Figure 3d), and they facilitate the translation between experimental changes in the optical parameters (objective position or ETL focal distance) to the actual imaging depth (Figure 3d, right axis and depth labels in Figure 3a).

Then, we conducted a set of experiments to explore the imaging performance of the new system. First, we compared the panretinal fluorescent angiograms in living mice that were acquired immediately following the injection using both our two-photon system and onephoton TEFI system ${ }^{8}$ (Figure $4 \mathrm{a}$, left). In contrast to the wide FOV images provided by the one-photon system, the angiograms obtained by the two-photon microscope have a limited FOV, but they have a higher lateral resolution and optical sectioning. The two-photon images allow the large blood vessels of the optic disc to be easily differentiated, and their relative positions could be determined, whereas only a few of the small blood vessels were visible compared with the one-photon image. Additionally, these blood vessels were displayed at a higher resolution. The optical sectioning was further demonstrated in the two-photon images that were obtained hours after the fluorescein injection (Figure $4 \mathrm{a}$, right) in which the strong out-of-focus signal from the choroidal vasculature did not prevent the ability to distinguish the small vessels, which were completely obscured in a reference late image acquired by the wide field (onephoton) system (Figure 4a, right).

Next, we tested whether the system can provide cellular-resolved images by imaging retinal cells that express GCaMP3. Transduced cells were clearly visualized in the microscopic images, and by acquiring a subsequent fluorescence angiogram, their location relative to the retinal vasculature was visualized (Figure $4 \mathrm{~b}$, vasculature shown in false colors). Using the doughnut-shaped cell images (Figure $4 \mathrm{~b}$ inset), which constitute the finest features visualized, we attempted to determine a rough 

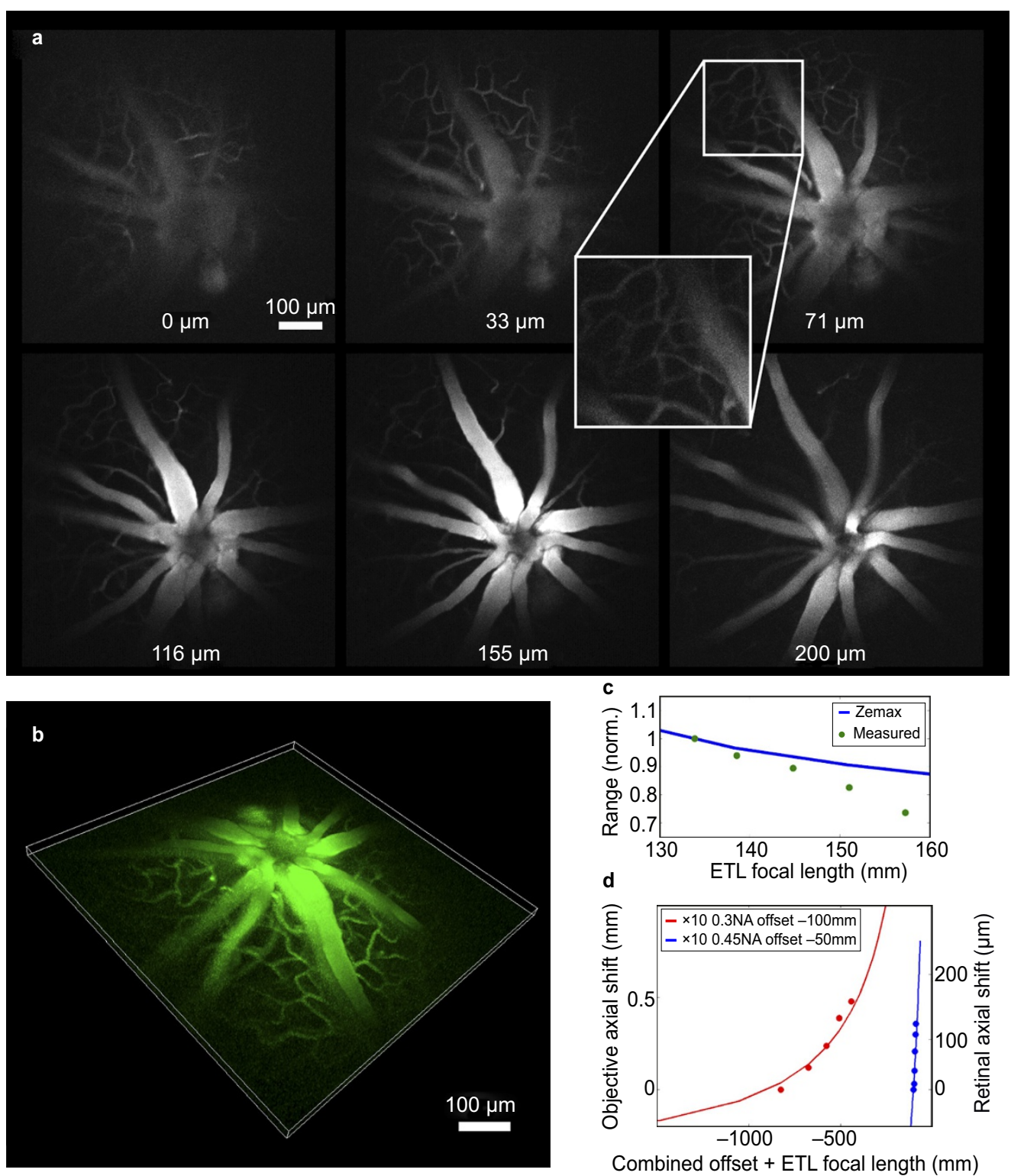

Figure 3 Two-photon mouse fluorescence angiography characteristics. (a) Two-photon optic disc images that were acquired at different ETL focal lengths; the microscope objective lens was kept static (average over 50 frames acquired at $1 \mathrm{fps}$ ). The marked axial shifts were calculated from the model. Inset: enlargement of the 71- $\mu \mathrm{m}$ image. (b) 3D reconstruction of multiple retinal planes. (c) The normalized effective measured FOV from the sections in A in comparison with the shift in the lateral scan range. (d) Measured (dots) vs. predicted (paraxial model, solid lines) axial shift as a function of combined offset and ETL focal length for the 0.3NA (red, $-100 \mathrm{~mm} \mathrm{CO}$ ) and $0.45 \mathrm{NA}$ (blue, $-50 \mathrm{~mm} \mathrm{CO}$ ) objectives. Left axis: the objective lens axial shift that was needed to refocus the image. Right axis: the transformation to the model-calculated retinal coordinates (blue retinal shifts; red are $2 \%$ smaller).

estimate for the lateral imaging resolution. The cross-sectional widths were evaluated for an average 'doughnut' ( $n=7$ cells, full-width at halfmaximum $(\mathrm{FWHM})=5.2 \mu \mathrm{m})$. To correct for the dimensions of the underlying cellular features, we performed the same calculation over a matching sample of GCaMP3-expressing cells that were imaged in an isolated retina in vitro and in high-resolution $(n=7$, FWHM $=4.0)$. From these values, we estimated that the lateral resolution upper bound is $\sim 3.5 \mu \mathrm{m}$ (see the section on 'Materials and methods').

Next, we tested whether it is possible to repeatedly image the same eye without damaging the retina or the ocular optics by repeating the imaging sessions across subsequent weeks in three of the animals. We did not observe damage (Figure 4c), and the images of the fine vasculature at the imaging site were very similar.
Finally, we examined the system's ability to perform functional calcium imaging in vivo by recording the calcium dynamics from multiple retinal ganglion cells (RGCs) that express GCaMP6s in response to full-field flashes of light in the blue spectrum (Figure $5 \mathrm{a}$ and $5 b)$. The cells were sparsely labeled, and the FOVs were small and contained 4-11 clearly visualized cells (5 FOVs in $n=2$ animals). In 20 of the 38 imaged cells, we observed putative calcium response traces, which repeated for each stimulus. The characteristic traces and mean responses shown in Figure 5c, which show four representative cells from two different animals, depict large and robust stimulus-locked responses with minimal attenuation/bleaching over the duration of the imaging sessions, and when stimulations were repeated 5 min later. The decay half-lives $\left(t_{1 / 2}\right)$, calculated from the exponential 

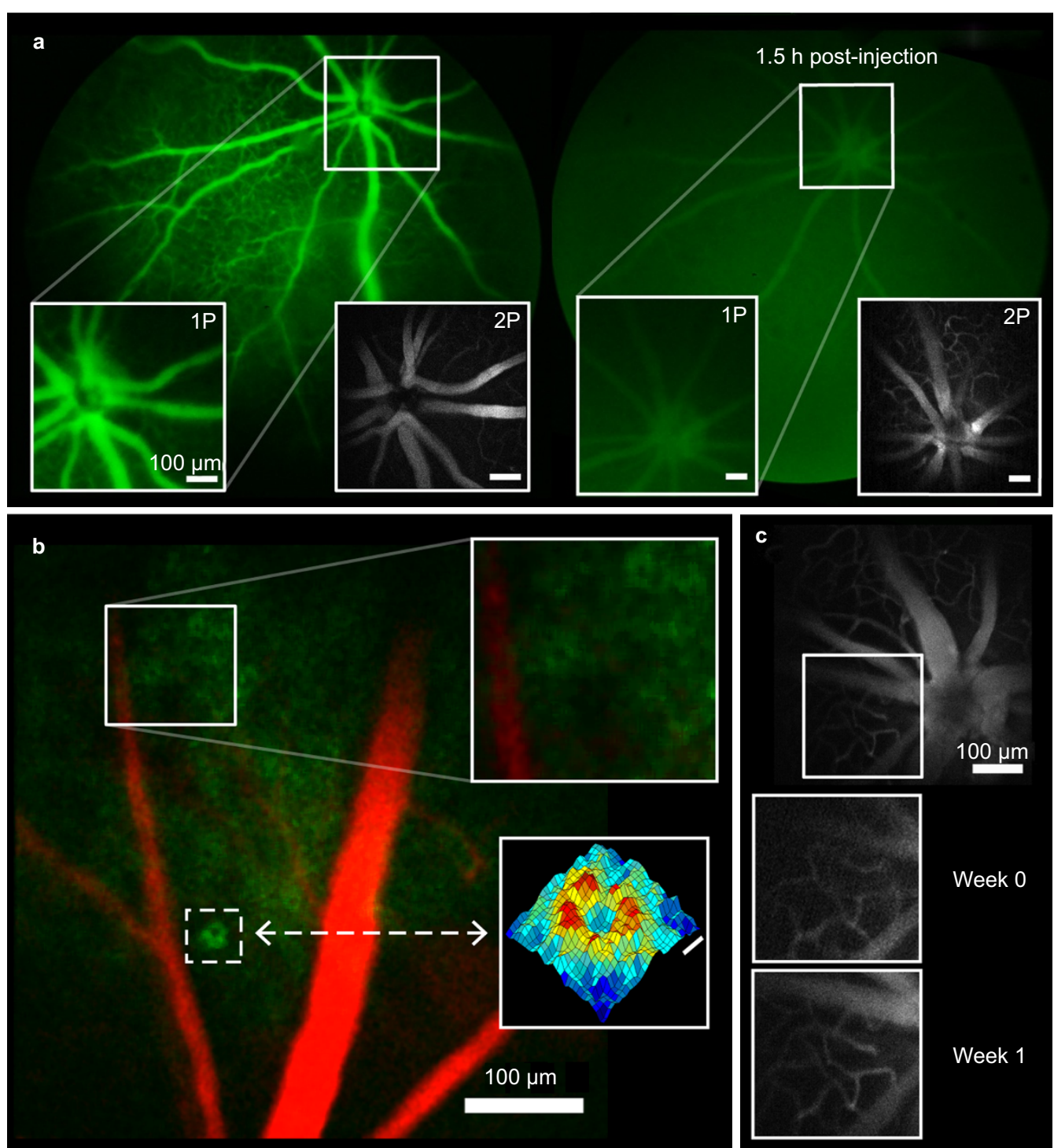

Figure 4 Imaging performance. (a) TEFI fluorescent angiograms (large images and corresponding enlargements, exposure time $=3 \mathrm{~s}$ ) vs. two-photon images of the same area (bottom right, average of 40 frames acquired at $1 \mathrm{fps}$ ). Images were acquired at $5 \mathrm{~min}$ (left) and $1.5 \mathrm{~h}$ (right) following the fluorescein injection (two different eyes shown). The strong out-of-focus signal from the choroidal vasculature that begins to appear at 20-min post-injection obscures the TEFI image, but it does not obscure the two-photon image. (b) False color image of the RGCs that express GCaMP3 (green) and blood vessels (red, averages of 120 and 50 frames, respectively). Insets: Enlargement of the area with multiple cells (top) and a surface plot of the average cell image (bottom, $n=7$, scale bar $=5 \mu \mathrm{m}$ ). (c) Repeated angiograms of the same retina that were obtained 1 week apart (averages of 30 and 50 frames, respectively).

fit of the average response, are very similar to the reported GCaMP6s decay half-lives for a multiple-spike response ${ }^{24}$, and they are qualitatively similar to the decay half-lives in RGC imaging studies using GCaMP $^{9,13}$. The half-life variation between cells (Figure $5 \mathrm{c}$, right) has also been observed in isolated mouse retinas (see, e.g., Figure 7 in Ref. 13). The initial response that is observed in Figure $5 \mathrm{c}$ preceding the visual flash stimuli was apparently due to the NIR imaging light, similar to what was reported by related in vitro studies ${ }^{10}$.

\section{CONCLUSIONS}

We demonstrated the in vivo acquisition of optically sectioned twophoton laser scanning microscopic mouse fundus images using an intuitive model-based design, which can be easily integrated into any existing two-photon microscope without requiring adaptive corrections. The insertion of tunable and concave offset lenses pro- vides the ability to image the retina at different depths without moving the optical elements near the eye at potentially $\mathrm{kHz}$ rates. The system provides detailed 3D micro-angiograms and cellularresolved images (of subcellular resolution). Using the paraxial model, which was validated by the ray-tracing Zemax model, it is possible to translate changes in the axial scan parameters to 'realworld' coordinates in the eye, which is not trivial as indicated by the 4.4 ratio between the axial focal shifts without and inside the eye. Our analysis provides an excellent prediction of the experimental results (Figure 2a, 2b and Figure $3 \mathrm{~d}$ ), and it suggests that the key hurdle toward this implementation is likely the paired selection of a combined offset lens with a large focal distance objective that can synergistically leverage the strong refraction that is already provided by the mouse crystalline lens to achieve a reliable focus on the retina. 
a
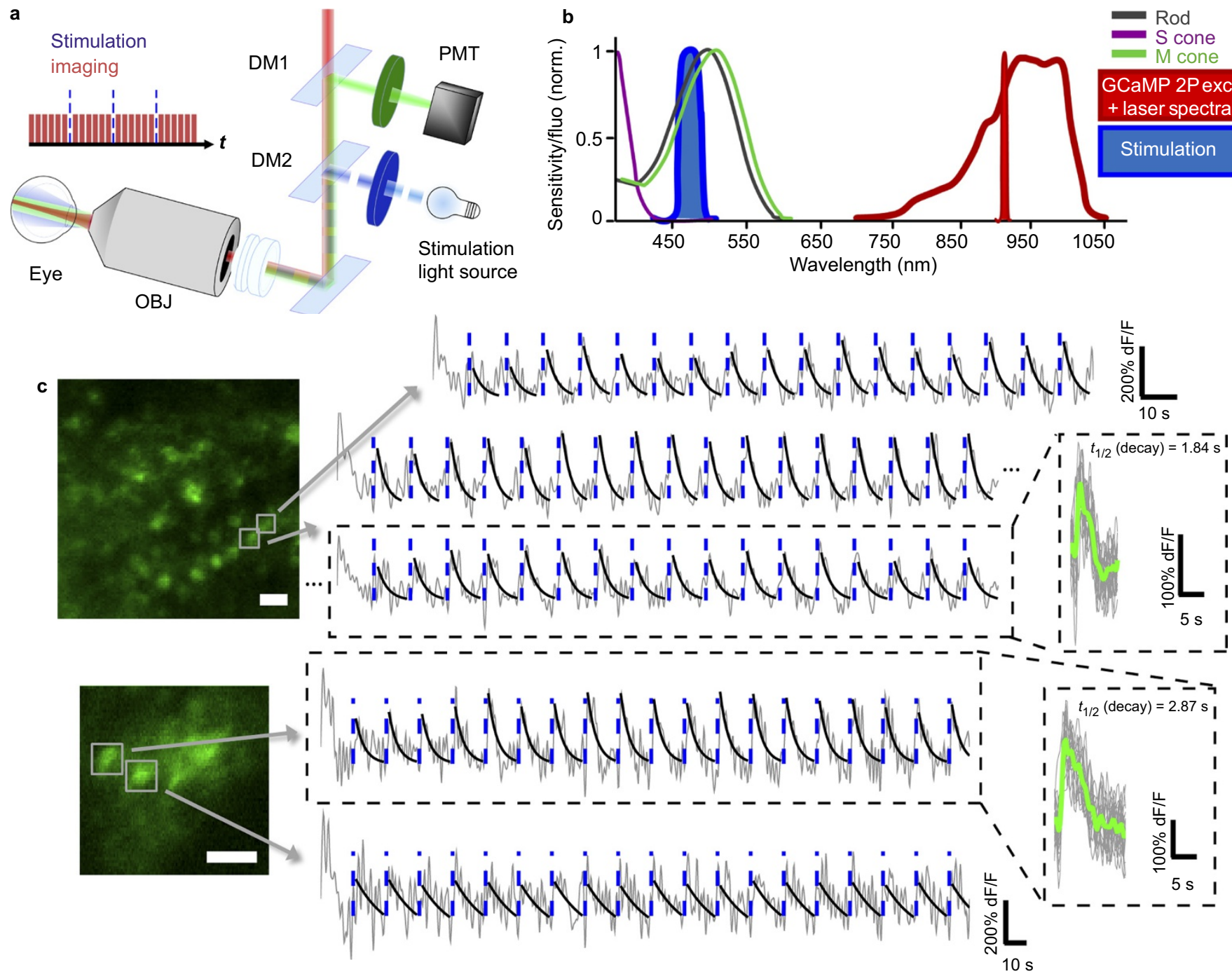

Figure 5 In vivo functional calcium imaging. (a) System schematic for the functional imaging experiments. Short pulses of blue light (10-20 ms) were flashed onto the retina as repeated scans of the FOV were acquired. (b) Spectra of the photoreceptor sensitivity of the mouse (adapted from Wang et al. ${ }^{26}$ ) and the GCaMP6 $2 \mathrm{P}$ excitation (adapted from Akerboom et al. ${ }^{27}$ ), which are superposed with the experimental stimulation and imaging wavelengths. (c) Representative calcium traces (gray) from four cells (marked in the average images on left) in response to the flashes of blue light (blue dashed lines) at 10- and 15-s intervals and acquired at 6.7 and $3.4 \mathrm{fps}$, as shown in the top and bottom panels, respectively. Top panel: the middle and bottom traces were acquired from the same cell and 5 min apart. The black lines represent the exponential decay functions that were fitted to the average response (marked by a green line on the right) and fitted to the magnitude of each single response. Image scale bars $=20 \mu \mathrm{m}$.

To our knowledge, the new system also provided the first demonstration of two-photon in vivo imaging of calcium responses in mice. Recently, the in vivo imaging of mouse RGC responses was demonstrated using single photon imaging ${ }^{9}$, which allowed only the recording of the responses to the UV flashes that were mediated by the $\mathrm{S}$-cones, as well as the $\mathrm{M}$-cone responses to the imaging light itself. In contrast, the spectral separation between the stimulation and NIR two-photon imaging wavelengths (Figure 5b) enables us to investigate the RGC responses mediated by both types of cones, as has been clearly demonstrated in isolated mouse retinas ${ }^{13,28}$. Instead of using the 1 - to 8 -sec timescale UV stimuli ${ }^{9}$, our ms-timescale and the visible stimuli provides opportunities for many new investigations of retinal physiology in its natural setting.

Comparing the two-photon and TEFI imaging results (Figure 4a) reveals that while the one-photon system suffers from low lateral and axial resolution because of its relatively low effective NA, the two-photon imaging system offers an improved lateral resolution and sectioning, and it effectively enables fluorescence angiography over much longer durations. It is also important to compare our correction-free two-photon approach with the recently introduced two-photon AO scanning laser ophthalmoscope (2PAOSLO) techniques ${ }^{17,18}$. Both the 2 PAOSLO systems use deformable mirror-based AO systems, but they have different compensation schemes and a different resulting complexity based on either a direct measurement of the phase distortion ${ }^{17}$ or on wavefront coding ${ }^{18}$ (which optimizes the resulting intensity of the images). In both cases, the pre-imaging time required for obtaining an optimal deformable mirror shape is considerable - as long as 4-6 $\min ^{18}$. The 2PAOSLO systems utilize air objectives and hard contact lenses to cancel the refractive power of the cornea, whereas our solution is based on the more customary water-dipping approaches. One benefit of our approach is that it allows for simple integration of accessory optical systems, such as photostimulation ${ }^{29}$, 
photo-coagulation $^{30}$, and optical coherence tomography (OCT), ${ }^{31}$ because they can be seamlessly combined into the same optical path. An important potential advantage of the 2PAOSLO approach is its image resolution; however, the resolutions cannot currently be compared directly because no lateral resolution estimates have been reported for the 2PAOSLO systems. Although we have only provided a model-based estimate of the possible resolutions $(\sim 1 \mu \mathrm{m})$ and an upper bound resolution measurement $(<3.5 \mu \mathrm{m}$, limited by the lack of smaller structures), we anticipate that the resolution of the system is close to these values and is in a range that is superior to the best results reported using non-AO single photon SLOs (FWHM 4.9-10 $\mu \mathrm{m}^{4,5}$ ). Qualitatively, the RGC images presented by Palczewska et al. ${ }^{18}$ (Figure 2d in Ref. 18) appear to have comparable quality to those shown in Figure $4 \mathrm{~b}$, and the average laser excitation power ranges applied through the pupil in all three of these studies are very similar (6-10 $\mathrm{mW}$ ). Thus, the newly demonstrated ability to perform correction-free high-quality imaging is an important baseline for $\mathrm{AO}$ approaches, and it will be important for future 2PAOSLO studies to examine their relative advantages in detail. In contrast to AO systems, our solution is a simple and inexpensive add-on that can be easily integrated into the majority of currently deployed two-photon microscopes, and it can be operated by a non-specialist.

Despite its simplicity, our approach has several potential pitfalls that require further consideration. First, to achieve simple analytical expressions for the dependence of the focal plane on the system design parameters, we utilized a paraxial model, which is expected to break down under large-angle conditions. However, this approach is retrospectively justified for the relatively small angles of the low-NA objectives that our analysis identified as suitable, as well as by the excellent agreement with the complementary and more general ray-tracing model (used exclusively for the PSF analysis). Second, the use of an ETL for axial scanning leads to variations in the imaging properties, including the lateral scan range/FOV and resolution, whose basic trends were similar to those found in Grewe et al. ${ }^{21}$ (but they were compounded by the eye aberrations). Despite these variations, we have demonstrated the ability to acquire high resolution retinal images with up to $\sim 1 \mathrm{~mm}$ side lengths over an axial range of $200 \mu \mathrm{m}$, which is equivalent to the thickness of the mouse retina ${ }^{22}$ (Figure $3 \mathrm{a}$ ). A related major common concern when using ETLs is with regard to their thermal drift profile. However, we did not encounter significant drifts in the timescale of the imaging that we used; moreover, recent device upgrades now provide automatic thermal drift compensation. Finally, we must address the question of safety in this new mode of imaging, which utilizes intense pulses of NIR lasers to illuminate the tissues of interest. By calculating the ANSI maximum permissible exposure (MPE, derived originally for human retinas) ${ }^{32}$, it is possible to estimate the safety limits for two-photon imaging of the mouse retina $(\sim 2.5$ $\mathrm{mW}$ for our scan parameters), and we were able to obtain good quality fluorescein angiograms using comparable power levels (3 mW). Importantly, even at the higher power levels used in this work ( $10 \mathrm{~mW}$ or $\sim 4$ times higher than the calculated MPE), the examination of the retina following two-photon imaging using both the TEFI and two-photon systems did not produce any apparent retinal damage $(n=6)$.

Our system provides new avenues for imaging neuronal retinal activity in response to natural, and potentially artificial-optogenetic ${ }^{29}$, visual stimuli within the visible spectrum using functional calcium imaging. This capacity is enabled by the use of wavelengths whose retinal sensitivity are orders of magnitude weaker in comparison to visible wavelengths ${ }^{17}$, although it is important to note that both direct and indirect retinal excitation does occur during the two-photon imaging $^{10,13}$. Moreover, the simplicity and versatility of this new system should facilitate the simple integration with other label-free imaging modalities, including OCT and intrinsic signal fundus imaging. Therefore, our correction-free approach can serve as a basis for new applications in retinal structural and functional imaging using two-photon fluorescence and related imaging ${ }^{33}$ and therapeutic modalities $^{34}$.

\section{ACKNOWLEDGEMENTS}

The authors thank Nizan Meitav, Adel Zeidan, Dvir Yelin, Yoreh Barak, Erez Ribak, Avner Shimron, Noam Cohen, and Tom Mayblum for their advice and assistance. Additionally, we thank Mark Blum (Optotune AG) for his assistance regarding the operation and modeling of the ETL; and Vivek Jayaraman, Douglas S. Kim, Loren L. Looger, and Karel Svoboda, from the GeneticallyEncoded Neuronal Indicator and Effector (GENIE) Project, Janelia Research Campus, Howard Hughes Medical Institute for permission to use GCaMP3 and GCaMP6. This research was supported by the European Research Council (ERC) under the European Union's Horizon 2020 research and innovation program, \#641171, by the Israel Science Foundation (ISF) \#1725/13, and by a Gutwirth Fellowship to A.S.

1 Webb RH, Hughes GW, Pomerantzeff O. Flying spot TV ophthalmoscope. Appl Opt 1980; 19: 2991-2997.

2 Roorda A, Romero-Borja F, Donnelly WJ, Queener H, Hebert TJ et al. Adaptive optics scanning laser ophthalmoscopy. Opt Express 2002; 10: 405-412.

3 Geng Y, Dubra A, Yin L, Merigan WH, Sharma R et al. Adaptive optics retinal imaging in the living mouse eye. Biomed Opt Express 2012; 3: 715-734.

4 Leung CK, Weinreb RN, Li ZW, Liu S, Lindsey JD et al. Long-term in vivo imaging and measurement of dendritic shrinkage of retinal ganglion cells. Invest Ophthalmol Vis Sci 2011; 52: 1539-1547.

5 Zhang P, Zam A, Pugh EN, Zawadzki R. Evaluation of state-of-the-art imaging systems for in vivo monitoring of retinal structure in mice: current capabilities and limitations. Proc SPIE 2014; 8930: 893005.

6 Kezic JM, Chen X, Rakoczy EP, McMenamin PG. The effects of age and $\mathrm{Cx}_{3} \mathrm{cr} 1$ deficiency on retinal microglia in the Ins $2^{\text {Akita }}$ diabetic mouse. Invest Ophthalmol Vis Sci 2013; 54: 854-863.

7 Paques M, Guyomard JL, Simonutti M, Roux MJ, Picaud S et al. Panretinal, highresolution color photography of the mouse fundus. Invest Ophthalmol Vis Sci 2007; 48: 2769-2774.

8 Schejter A, Tsur L, Farah N, Reutsky-Gefen I, Falick Y et al. Cellular resolution panretinal imaging of optogenetic probes using a simple funduscope. Trans Vis Sci Tech 2012; 1: 4.

9 Yin L, Geng Y, Osakada F, Sharma R, Cetin AH et al. Imaging light responses of retinal ganglion cells in the living mouse eye. J Neurophysiol 2013; 109: 2415-2421.

10 Denk W, Detwiler PB. Optical recording of light-evoked calcium signals in the functionally intact retina. Proc Natl Acad Sci USA 1999; 96: 7035-7040.

11 Euler T, Detwiler PB, Denk W. Directionally selective calcium signals in dendrites of starburst amacrine cells. Nature 2002; 418: 845-852.

12 Dreosti $\mathrm{E}$, Esposti $\mathrm{F}$, Baden T, Lagnado L. In vivo evidence that retinal bipolar cells generate spikes modulated by light. Nat Neurosci 2011; 14: 951-952.

13 Borghuis BG, Tian L, Xu Y, Nikonov SS, Vardi N et al. Imaging light responses of targeted neuron populations in the rodent retina. J Neurosci 2011; 31: 28552867.

14 Imanishi Y, Batten ML, Piston DW, Baehr W, Palczewski K. Noninvasive two-photon imaging reveals retinyl ester storage structures in the eye. J Cell Biol2004; 164: 373 383.

15 Gualda EJ, Bueno JM, Artal P. Wavefront optimized nonlinear microscopy of ex vivo human retinas. J Biomed Opt 2010; 15: 026007.

16 Gibson EA, Masihzadeh O, Lei TC, Ammar DA, Kahook MY. Multiphoton microscopy for ophthalmic imaging. J Ophthalmol 2011; 2011 : 870879.

17 Sharma R, Yin L, Geng Y, Merigan WH, Palczewska G et al. In vivo two-photon imaging of the mouse retina. Biomed Opt Express 2013; 4: 1285-1293.

18 Palczewska G, Dong Z, Golczak M, Hunter JJ, Williams DR et al. Noninvasive twophoton microscopy imaging of mouse retina and retinal pigment epithelium through the pupil of the eye. Nat Med 2014; 20: 785-789.

19 Geng Y, Schery LA, Sharma R, Dubra A, Ahmad K et al. Optical properties of the mouse eye. Biomed Opt Express 2011; 2: 717-738.

20 Schejter A, Farah N, Shoham S. Two-photon in vivo imaging of retinal microstructures. Proc SPIE 2014; 8948: 894824.

21 Grewe BF, Voigt FF, van't Hoff M, Helmchen F. Fast two-layer two-photon imaging of neuronal cell populations using an electrically tunable lens. Biomed Opt Express 2011; 2: 2035-2046. 
22 Remtulla S, Hallett PE. A schematic eye for the mouse, and comparisons with the rat. Vision Res 1985; 25: 21-31.

23 Tian L, Hires SA, Mao T, Huber D, Chiappe ME et al. Imaging neural activity in worms, flies and mice with improved GCaMP calcium indicators. Nat Methods 2009; 6: 875 881.

24 Chen TW, Wardill TJ, Sun Y, Pulver SR, Renninger SL et al. Ultrasensitive fluorescent proteins for imaging neuronal activity. Nature 2013; 499: 295-300.

25 Pologruto TA, Sabatini BL, Svoboda K. Scanlmage: flexible software for operating laser scanning microscopes. Biomed Eng Online 2003; 2: 13.

26 Wang YV, Weick M, Demb JB. Spectral and temporal sensitivity of cone-mediated responses in mouse retinal ganglion cells. J Neurosci 2011; 31: 7670-7681.

27 Akerboom J, Chen TW, Wardill TJ, Tian L, Marvin JS et al. Optimization of a GCaMP calcium indicator for neural activity imaging. J Neurosci 2012; 32: 13819-13840.

28 Briggman KL, Euler T. Bulk electroporation and population calcium imaging in the adult mammalian retina. J Neurophysiol 2011; 105: 2601-2609.

29 Reutsky-Gefen I, Golan L, Farah N, Schejter A, Tsur L et al. Holographic optogenetic stimulation of patterned neuronal activity for vision restoration. Nat Commun 2013; 4: 1509.

30 Paulus YM, Jain A, Nomoto H, Sramek C, Gariano RF et al. Selective retinal therapy with microsecond exposures using a continuous line scanning laser. Retina 2011; 31 380-388.
31 Liu JJ, Grulkowski I, Kraus MF, Potsaid B, Lu CD et al. In vivo imaging of the rodent eye with swept source/Fourier domain OCT. Biomed Opt Express 2013; 4: 351-353.

32 Delori FC, Webb RH, Sliney $\mathrm{DH}$. Maximum permissible exposures for ocular safety (ANSI 2000), with emphasis on ophthalmic devices. J Opt Soc Am A 2007; 24: 1250-1265.

33 Klemm M, Dietzel A, Haueisen J, Nagel E, Hammer M et al. Repeatability of autofluorescence lifetime imaging at the human fundus in healthy volunteers. Curr Eye Res 2013; 38: 793-801.

34 Manevitch Z, Lewis A, Levy C, Zeira E, Banin E et al. Subsurface femtosecond tissue alteration: selectively photobleaching macular degeneration pigments in near retinal contact. J Phys Chem B 2012; 116: 6945-6951.

(c) (-) $\odot$ This work is licensed under a Creative Commons Attribution-

BY NC ND NonCommercial-NoDerivs 4.0 Unported License. The images or other third party material in this article are included in the article's Creative Commons license, unless indicated otherwise in the credit line; if the material is not included under the Creative Commons license, users will need to obtain permission from the license holder to reproduce the material. To view a copy of this license, visit http://creativecommons.org/licenses/ by-nc-nd/4.0/

Supplementary information for this article can be found on the Light: Science \& Applications' website (http://www.nature.com/lsa/). 